\title{
Survey of Image Analysis Methods Applied to Consumer Foods
}

\author{
Nicolas Piche ${ }^{1}$, Emimal Jabason ${ }^{1}$, Mike Marsh ${ }^{2}$ and Almut H. Vollmer ${ }^{3}$
}

1. Object Research Systems. Montreal, Canada.

2. Object Research Systems. Denver, CO.

3. Dept of Nutrition, Dietetics and Food Sciences. Utah State University. Logan, UT.

Imaging methods and associated image processing techniques are frequently described in applications for materials and life sciences. Contrast mechanisms and key research questions often diverge greatly between the unrelated disciplines. There is, however, great overlap in the software tools required to transform the fundamentally different images into quantitative findings, and this is seen clearly in food imaging, where samples exhibit both material and life science properties. Here we use two very different foods to illustrate image analysis applications in the domain of consumer food microstructure characterization, and we rely on the commercial software Dragonfly as a single unified platform for image analysis and visualization. First we consider feta cheese samples on a length scale of $50 \mu \mathrm{m}$, where samples were imaged by TEM following various experimental treatments. Second, we look at multi-scale x-ray microscopy imaging of a chocolate wafer bar, imaged on scales spanning micrometers to millimeters.

Our first sample is feta cheese stained with heavy metal contrast agents and imaged in a JEOL JEM $1400 \mathrm{TEM}$ at a nominal magnification of 500x, with an approximate horizontal field of view of $50 \mu \mathrm{m}$ and a pixel size of approximately $12.5 \mathrm{~nm}$; experimental conditions have been described elsewhere [1]. TEM image contrast clearly shows three phases, but intensities greatly overlap in the image histogram, making simple intensity thresholding inadequate to properly resolve different phases. We apply the Dragonfly Segmentation Trainer machine-learning classifier [2] to segment the image into protein, fat, and matrix phases. Our segmentation relies on a Random Forest classifier engine, applied to feature vectors built from image processing executed on the image in a region-based fashion, including vector components derived from filter banks of many filters including Gabor and Neighbors filters. Following segmentation, we measured the area fraction of each phase, and found that results vary for different treatments. We show here (Figure 1) the area fractions for each phase in a representative micrograph.

Our second food sample was a Hershey's KitKat chocolate covered wafer bar. With no sample preparation other than unwrapping, we imaged this sample on an Xradia Versa $520 \mathrm{x}$-ray microscope with a field-of-view of $20 \mathrm{~mm}$ (in each dimension) and a pixel size of approximately $20.0 \mu \mathrm{m} / \mathrm{pixel}$; at this scale, we can clearly see multiple stacked wafer layers intercalated with chocolate. We performed subsequent imaging at higher resolution $(3.55 \mu \mathrm{m} / \mathrm{pixel})$ to reconstruct the interior of the bar (without any further sample preparation); at this scale we, can see the microstructure which clearly exhibits three phases for the chocolate, whereas the wafer appears to be a closed pore foam with nonuniform bubbles with a highly heterogeneous wall-thickness and bubble size distribution.

Inspection at the coarse scale reveals first that the surrounding chocolate has very different microstructure from the chocolate layers sandwiching the wafers. We observe an interlayer wafer spacing of approximately $3 \mathrm{~mm}$, and find that the wafer has different nonplanar lattice patterns on its top and bottom surfaces. We further used systematic cross-sectional measurements to interrogate sample uniformity along the axis of the wafer, which shows surprising heterogeneity. 
Fine scale imaging showed the interior chocolate to have three phases: matrix, grains, and air pockets, all of which we segmented again with a machine learning classifier. We segmented the two phases of the wafer to find the pore size distribution and volume fractions of the foam. The wafer foam was visualized by color-coding the rendering according to local wall thickness.

These two examples show that image analysis can characterize numerous distinctive microstructure properties. Mapping these microscale findings onto commercially competitive features (e.g. flavor, texture, creaminess, etc.) provides a great opportunity for food formulation scientists to relate those properties directly to the microstructure for directed experimentation to optimize formulation for superior food products.

References:

[1] A H Vollmer et al, Microsc Microanal 23(1) (2017), p. 1128.

[2] N Piche et al, Microsc Microanal 23(1) (2017), p. 246.

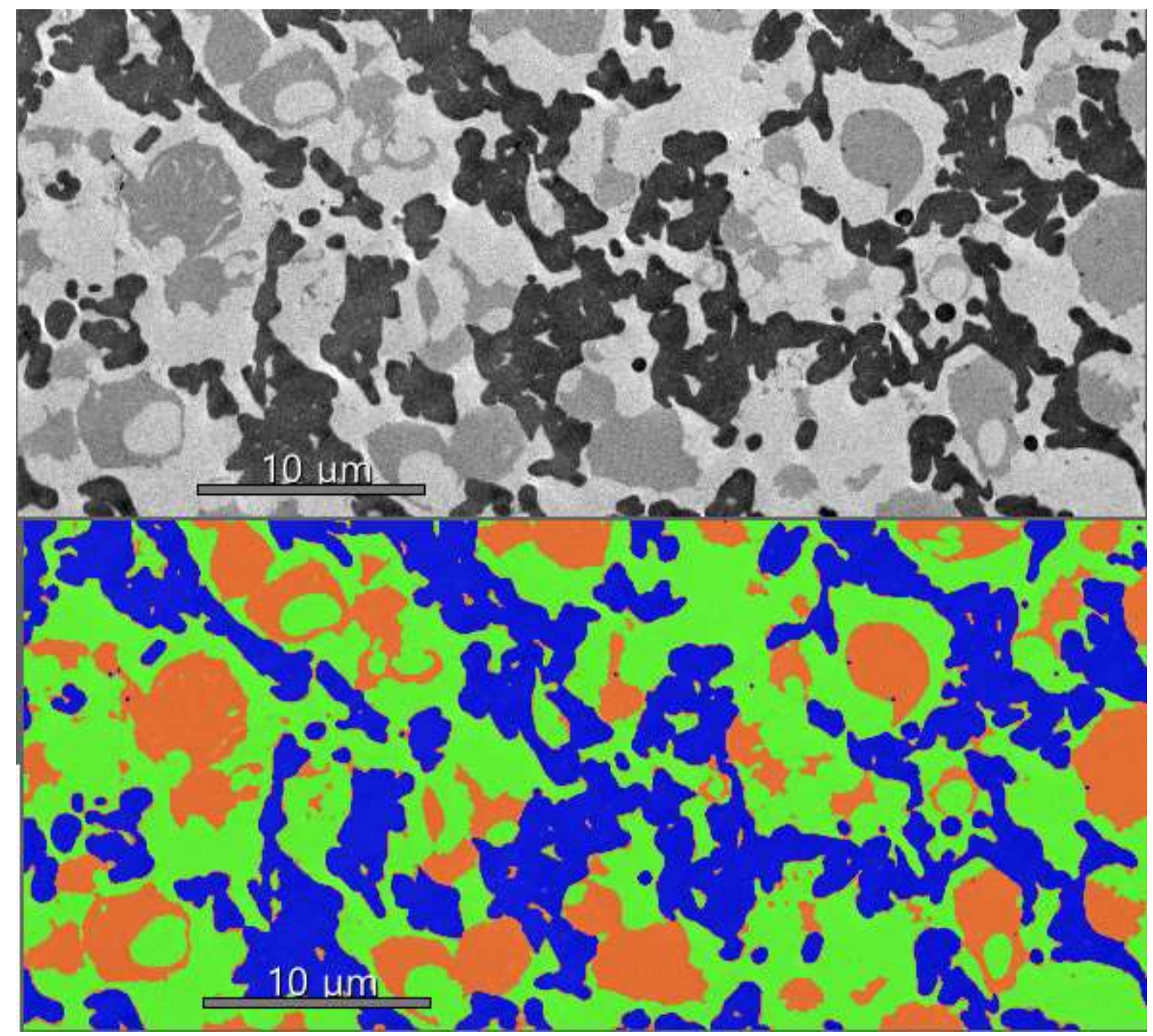

Figure 1. Feta cheese sample TEM image (top) and segmented image (bottom), where protein matrix (blue), fat (orange), serum phase (green) fractions are observed to be $32.7 \%, 23.5 \%$, and $43.7 \%$ respectively. 